\title{
Application of Particle Swarm Optimization Algorithm
}

\section{to Electric Power Line Overhaul Plan}

\author{
Jia Liu, Yang Li (Corresponding author) \& Liqun Gao \\ School of Information Science and Technology \\ Northeastern University \\ 3-11 Wen Hua Road, Shenyang 110004, China \\ E-mail: liyang@ise.neu.edu.cn
}

\begin{abstract}
Electric power line overhaul plan is an important issue on power system and engineering practice. As particle swarm optimization is to be a new intelligent algorithm. It is gradually applied into power system these years. This paper provides a relative mathematical model to solve the problems in power line overhaul. Particle swarm optimization algorithm has advantages of less parameters setting and highly optimizing speed. Take these advantages; we make an optimal order of electric power line overhaul. It is proved that the application of particle swarm optimization algorithm to electric power line overhaul plan is feasible.
\end{abstract}

Keywords: Electric power line overhaul plan, Particle swarm algorithm, Optimization algorithm, Power system

\section{Introduction}

With rapid expand of power system scales and implementation of electricity market reformation. To make the power system operate on a security, economic, stable and reliable way becomes more important while the security and economic factors becomes more complex. With different objections of planning and operation in power system, the choice of different control variables and constraints may propose different optimization. It needs the help of optimal theory. Especially with extensive search on intelligent heuristic algorithm which applied on power system. It supports a new method to solve the relative question in power system.

Particle Swarm Optimization (PSO) is one of the latest evolutionary optimization techniques developed by Eberhart and Kennedy PSO concept is based on a metaphor of social interaction such as bird flocking. This algorithm provides a robust, parallel processing approach to obtain the global best solution with higher probability. This method is easy to implement and fast to convergence. As its intelligence background, it can apply both to science research and engineering program. It aroused widespread interest as soon as it is proposed, and obtain a large number of achievements in a short time. It is used in many fields such as function optimization, neural network design, pattern recognition, signal processing, and robot technology and so on.

Electric power line overhaul is multi-objective and nonlinear complex optimization. The main task is to find out best overhaul programmed in order to reduce maintenance costs when the power grid happen to be failure. Electric power line overhaul plan is complex optimization for its time-varying, discretion, nonlinear and randomness. So far, this issue has not been satisfactorily resolved.

PSO in the application of power system is on a late start. It shows broad prospects in the field of power system and has begun to attract the attention of the scientist who works on power system. Especially with the establishment and improvement in electricity market, how to take advantages of PSO algorithm to solve the problem of power system will become hot.

\section{The principle of PSO}

PSO is an evolutionary algorithm which is initialized with a population of random solutions and searches for optima by updating generations. Each individual or potential solution, named particle, flies in the dimensional problem space with a velocity which is dynamically adjusted according to the flying experiences of its own and its colleagues. The particle evaluate their positions relative a global fitness during each generation and share memories of their best positions, and use those memories to adjust their own velocities and subsequent positions.

Suppose that the searching space is $D$-dimensional and $m$ particles from the colony. The $i$ th particle represents a $D$-dimensional vector $X_{i}(t)=\left(x_{i 1}(t), x_{i 2}(t), \ldots, x_{i d}(t)\right)$ in the searching space, the $i$ th particle's "flying" velocity also a 
D-dimensional vector, denoted as $V_{i}(t)=\left(v_{i 1}(t), v_{i 2}(t), \ldots, v_{i d}(t)\right)$.Denote the best position of the $i$ th particle as $P_{i}(t)=\left(p_{i 1}(t), p_{i 2}(t), \ldots, p_{i d}(t)\right)$, and best position of the colony as $P_{g}(t)=\left(p_{g 1}(t), p_{g 2}(t), \ldots, p_{g d}(t)\right)$

The particles velocities and position are updated by the following equations:

$$
\begin{gathered}
V_{i k}(t+1)=w v_{i k}(t)+c_{1} \operatorname{rand} 1\left(p_{i k}(t)-x_{i k}(t)\right)+c_{2} \operatorname{rand} 2\left(p_{g k}(t)-x_{i k}(t)\right) \\
X_{i k}(t+1)=x_{i k}(t)+v_{i k}(t+1)
\end{gathered}
$$

$w$ is the weight parameter, it has a global search ability when $\mathrm{w}$ is large. It tends to be a local search when $w$ is small. Generally, $w=0.9$ in at first and decrease to 0.4 along with the increasing of generation. $c_{1}, \mathrm{c}_{2}$ are learning factors, rand and $\operatorname{rand}_{2}$ are random numbers between $(0,1), \mathrm{i}=1,2, \ldots, \mathrm{m} ; \mathrm{k}=1,2, \ldots, \mathrm{d}$. A particle's velocity in each dimension is clamped to a maximum velocity $V_{\mathrm{i}}$ and is set to a certain fraction of the range of the search space in each dimension.

\section{Model of Electric Power Line Overhaul Plan}

\subsection{Knapsack problem}

Knapsack problem is a typical optimization in operations research. It is applied to budgetary controlling, item selecting, materials cutting and goods loading and searched as sub problems. With the development of network, public-key system of knapsack plays an important role in public-key design .Knapsack problem can be described as follows:

$$
\begin{gathered}
\max f\left(x_{1}, x_{2}, \ldots, x_{n}\right)=\sum_{j=1}^{n} c_{j} x_{j} \quad \mathrm{j}=1,2 \ldots \mathrm{n} \\
\sum_{j=1}^{n} a_{i j} x_{j} \leq b_{j} \quad \mathrm{i}=1,2 \ldots \mathrm{m} ; x_{j} \in\{0,1\}
\end{gathered}
$$

Where $\mathrm{n}$ is the number of objects, $\mathrm{m}$ is the number of resource, $c_{\mathrm{j}}$ is profit of object $j, b_{\mathrm{i}}$ is a budget for resource, $a_{\mathrm{ij}}$ is consumes of resource $i, x_{\mathrm{j}}$ is a $0 / 1$ decision variables (when the object $j$ is chosen, $x_{\mathrm{j}}=1$, else $x_{\mathrm{j}}=0$ ).

$\mathrm{KP}$ can be regarded as a resource allocation problem, where we have $m$ resources $a_{\mathrm{ij}}(\mathrm{i}=1,2, \ldots, \mathrm{m})$ and $n$ objects $\mathrm{j}(\mathrm{j}=1,2, \ldots, \mathrm{n})$. Each resource $\mathrm{i}$ has a budget $b_{\mathrm{i}}$, each object $\mathrm{j}$ has a profit $c_{\mathrm{j}}$ and consumes $a_{\mathrm{ij}}$ of resource $\mathrm{i}$. The problem is to maximize the profit within a limited budget. Knapsack problem can be derived from a series of related optimization problem.

Knapsack problem has wide applications in practice. Much organic combination with simple structure makes complex structure. The way to solve a complex problem can be easy when some simple problem can be solved. In the solution of complex optimization, Knapsack problem is always as a sub problem. It can get a better solution in optimization from the improvement of Knapsack problem.

\subsection{Solution knapsack problem based on particle swarm optimization}

In $0 / 1$ knapsack problem, the variable $i$ is set to be 1 if object $i$ is placed in the knapsack. According to genetic algorithm, we can regard $x_{1}, x_{2}, \ldots, x_{n}$ as binary variables. It can convert to decimal as:

$$
y_{1}=x_{1} \times 2^{n-1}+x_{2} \times 2^{n-2}+\ldots+x_{n}
$$

It can also be pressed as two integers:

$$
\begin{aligned}
& y_{1}=x_{1} \times^{n / 2-1}+x_{2} \times 2^{n / 2-2}+\ldots+x_{n / 2} \\
& y_{2}=x_{n / 2+1} \times 2^{n / 2-1}+x_{n / 2+2} \times 2^{n / 2+2}+\ldots+x_{n}
\end{aligned}
$$

We can decide the number

The solution of knapsack problem mapping from its solution space to some discrete points on one (or more )lines in Euclidean space. Next we can get the solution by using PSO. But we should note that the solution space is not continuous, so we need rounding operation in position computing. Through the test, we can get a better solution using this algorithm.

Generate 100 random numbers in range [0,1], which represent the value of weight. Take an average value when the test runs 10 times.

\subsection{The combination of model and the actual}

With the constraints in electric power line overhaul and the similarities between workload and working hours, we can apply the model of $0 / 1$ knapsack problem to electric power line overhaul.

In order to reach the optimization objectives, we do some changes in the initial model of knapsack problem:

$$
J=\min \left(b_{i}-\sum_{j=1}^{n} a_{j} x_{j}\right) \quad j=1,2, \ldots, n
$$




$$
\text { s.t. } \sum a_{j} x_{j} \leq b_{i} \quad j=1,2, \ldots, n \quad i=1,2, \ldots, m
$$

$b_{i}$ represents workload in ith month, $x_{j}$ is a $0 / 1$ decision variables(when the task is chosen, $x_{j}=1$, else $x_{j}=0$ ). $a_{j}$ reprents the workload we need to finish ith task.

Formula (9) gives the constraints of electric power line overhaul to make actual workload less than that in plan. Formula (8) is a definition of objection function. J represents a difference between actual workload and planning workload per month.

We should consider the arrangements in actual work condition. For example, we assigned little task in cold winter like January to April. Otherwise we assigned more tasks in May and June.

The correspondence between original and improved model is that: there are three parameters in knapsack problem; they are size of knapsack, weight of object, value of object. In improved model, the weight and value of an object represent the workload. The sizes of knapsack represent the planning workload.

\section{Experimental results}

Though Matlab is widely used in simulation, the proposed PSO algorithm for electric power line overhaul plan was implemented in Matlab language. In main procedures of knapsack problem, we choose the data in March to June. The actual assignments are 100. We assigned little in March and April and a little more in May and June. The actual for every task is:

$\begin{array}{rrrrrrrrrr}\mathrm{W}=3 & 2 & 3 & 5 & 7 & 8 & 6 & 4 & 5 & 3 \\ 4 & 8 & 5 & 1 & 7 & 3 & 9 & 1 & 2 & 8 \\ 4 & 7 & 3 & 2 & 6 & 8 & 1 & 7 & 5 & 6 \\ 10 & 2 & 9 & 8 & 1 & 2 & 3 & 4 & 3 & 1 \\ 6 & 4 & 7 & 5 & 8 & 4 & 3 & 1 & 5 & 4 \\ 2 & 4 & 7 & 5 & 2 & 3 & 6 & 7 & 3 & 4 \\ 4 & 6 & 8 & 3 & 4 & 7 & 5 & 1 & 9 & 3 \\ 2 & 9 & 4 & 7 & 5 & 6 & 1 & 4 & 3 & 7 \\ 2 & 8 & 3 & 4 & 2 & 1 & 6 & 4 & 2 & 3 \\ 1 & 8 & 7 & 4 & 3 & 4 & 6 & 8 & 2 & 4\end{array}$

The second step is to number the task:

\begin{tabular}{|c|c|c|c|c|c|c|c|c|c|c|}
\hline \multirow{10}{*}{ order $=$} & 1 & 2 & 3 & 4 & 5 & 6 & 7 & 8 & 9 & 10 \\
\hline & 11 & 12 & 13 & 14 & 15 & 16 & 17 & 18 & 19 & 20 \\
\hline & 21 & 22 & 23 & 24 & 25 & 26 & 27 & 28 & 29 & 30 \\
\hline & 31 & 32 & 33 & 34 & 35 & 36 & 37 & 38 & 39 & 40 \\
\hline & 41 & 42 & 43 & 44 & 45 & 46 & 47 & 48 & 49 & 50 \\
\hline & 51 & 52 & 53 & 54 & 55 & 56 & 57 & 58 & 59 & 60 \\
\hline & 61 & 62 & 63 & 64 & 65 & 66 & 67 & 68 & 69 & 70 \\
\hline & 71 & 72 & 73 & 74 & 75 & 76 & 77 & 78 & 79 & 80 \\
\hline & 81 & 82 & 83 & 84 & 85 & 86 & 87 & 88 & 89 & 90 \\
\hline & 91 & 92 & 93 & 94 & 95 & 96 & 97 & 98 & 99 & 100 \\
\hline
\end{tabular}

We can get the information about finished task and unfinished task by numbering the data.

The third step is to put the task which has been numbered into knapsack. Then get the best arrangement. The arrangements of each month are as follows:

$\begin{array}{cccccccccc}\mathrm{m} 1= & 7 & 8 & 9 & 14 & 16 & 18 & 19 & 22 & 23 \\ 24 & 33 & 35 & 37 & 38 & 39 & 42 & 45 & 49 & 50 \\ 52 & 60 & 62 & 64 & 65 & 66 & 77 & 79 & 81 & 83 \\ 84 & 88 & 90 & 100 & & & & & & \\ \mathrm{~m} 2=1 & 13 & 20 & 25 & 29 & 30 & 32 & 40 & 51 & 56 \\ 58 & 68 & 74 & 76 & 80 & 82 & 94 & 99 & & \end{array}$




$\begin{array}{rrrrrrrrrr}\mathrm{m} 3= & 10 & 11 & 12 & 15 & 26 & 27 & 31 & 41 & 43 \\ 44 & 48 & 54 & 55 & 61 & 69 & 71 & 75 & 85 & 86 \\ 91 & 97 & & & & & & & & \\ \mathrm{~m} 4= & 5 & 6 & 17 & 21 & 28 & 34 & 36 & 46 & 47 \\ 53 & 57 & 63 & 67 & 72 & 73 & 78 & 89 & 92 & 93 \\ 95 & 98 & & & & & & & & \end{array}$

Then deal with the data by improved knapsack problem model, we get:

The planning workload of March $=\mathrm{b} 1=150$

The actual workload of March $=136=49+3+4+3+4+8+5+4+4+4+6+7+5+6+1+3+2+3+4+4+3+4$

The planning workload of April $=\mathrm{b} 2=100$

The actual workload of April $=83=3+5+8+6+5+6+3+2+3+7+8+6+7+8+4+3+3$

The planning workload of May $=\mathrm{b} 3=120$

The actual workload of May $=100=3+4+8+8+1+10+6+7+5+1+5+2+2+9+4+5+3+7+7+2$

The planning workload of June $=\mathrm{b} 4=140$

The actual workload of June $=129=2+7+8+9+4+7+8+4+7+6+8+5+9+4+4+2+8+7+3+8+4+3+2$

According to the data above, the workload in each month all satisfy the constraints but the effect are different.

The value of object function in March $J_{1}=150-139=11$

The value of object function in April $J_{2}=100-83=17$

The value of object function in May $J_{3}=120-100=20$

The value of object function in June $J_{4}=150-129=21$

\section{Conclusion}

This paper noticed the importance of electric power line overhaul which has some problems in power system, then proposed a knapsack problem model. Use PSO algorithm to solve the problem and simulate with Matlab. The result shows the correctness and feasible to solve the problem using PSO algorithm.

\section{References}

Kennedy J, Eberhart R. (1995). Particle swarm optimization. Proceedings of IEEE Conference on Neural Networks. Perth, Australia, 4, 1942-1948.

Sensarma P S, Rahmani M. (2002). A comprehensive method for optimal expansion planning using particle swarm optimization. Proceedings of the IEEE Power Engineering Society Transmission and Distribution Conference. New York, USA, 1317-1322.

Fukuyama Y. (2002). Modern Heuristic Optimization Techniques with Applications to Power Systems. IEEE Power Engineering Society. $45-51$.

Pirlot P. M. (1996). General local search methods. European Journal of Operational Research. 92: 493-511.

Abe S. (1992). Solving inequality constrained combinational optimization problems by the Hopfield neural network. Neural Network. 5: 633-670.

FATIH M.A. (2003). Binary particle swarm optimization algorithm for lot sizing problem. Journal of Economic and Social Research. 5(2): 1-20. 